\title{
Fractal Geometry and Nonlinear Analysis in Medicine and Biology
}

\section{Editorial}

\section{The fractal geometry of the brain}

\author{
Antonio Di Ieva*1 \\ ${ }^{1}$ Macquarie University, Sydney, NSW, Australia
}

As well known, fractals have several applications in many fields, including the biomedical sciences and the neurosciences. The human brain, with its exquisite complexity, can be seen as a fractal object, and fractal analysis can be successfully applied to analyze its wide physiopathological spectrum and to describe its self-similar patterns, in both neuroanatomical architecture and neurophysiological timeseries.

Reminiscent of the milestone book, Mandelbrot's The Fractal Geometry of Nature, a recent multi-author collaboration, The Fractal Geometry of the Brain (Springer, New York, 2016) summarizes the state of the art fractal analysis in neuroscience.

Thanks to the extraordinary contributions of 58 experts worldwide (physicians, basic and clinical neuroscientists, computer scientists, mathematicians, psychologists, biologists, physicists and engineers amongst others) from 23 different countries, who have made personal contributions in the field, the book offers an holistic view on the most intriguing applications of fractal analysis to the neurosciences with a focus on current applications and future perspectives, limits, advantages and disadvantages. It is essentially for scientists, scholars, physicians and students of different disciplines interested in applying fractal-based computational models to the study of the brain. The book is mainly clinically oriented, offering suggestions for applying fractals "from bench to bedside", but also covers numerous research aspects. More than discoursing on sterile epistemological interpretations to define whether the brain is or is not a mathematical fractal object, the authors describe how computational fractal-based analysis can help in analyzing the marvelous and complex system called the brain in its wholeness from neurons and microglia cells to networks, and from normal time-series to pathological processes and diseases, all to give fractals the dignity of being deemed a useful biomarker for practical and clinical applications.
To achieve this aim, the book is uniquely organized into four sections, with each section briefly introduced by an editor's chapter that summarizes the topics described by the authors. In the first section, the laymen can find a snapshot of the "nuts and bolts" of fractal geometry while the experts can refresh their knowledge, and all can understand the applications of fractals to basic and clinical neurosciences. The second section then begins to investigate the brain as a fractal object in a hierarchical, multilevel analysis from neurons and microglia to the whole brain itself, with all its structural and functional complexity. The third section follows by building upon the second and summarizes applications of computational fractal-based analysis to the practical applications in the clinical neurosciences, moving sequentially through the different disciplines. No topic is omitted; indeed neurology and neurosurgery, neuroimaging, neuropathology and neurohistology, neurooncology, neuro-ophthalmology, neuropsychiatry, and even neuro-esthetics are the targets of the chapters. For people interested on how they may apply computational fractal-based neurosciences, the fourth section of the book illustrates software and computer science tools, also introducing some bioengineering-related aspects of fractal geometry, including fractal neuronal interfaces to future perspectives in artificial intelligence.

As introduced by an excellent foreword by Bruce West, the authors, who include very well known names in the fractal realm, such as Audrey Karperien, Herbert Jelinek, Michel Hofman, Francisco Esteban, Giorgio Bianciardi, amongst others, offer a very comprehensive excursus in the field, with the hopes of providing a stimulus that ignites new ideas in the open minds of out-of-the-box thinkers who are interested in the study and treatment of the brain from a novel and progressive perspective; an aim which appears to be widely shared by the FGNAMB journal.
Copyright: (C2016 Ieva AD. This is an open-access article distributed under the terms of the Creative Commons Attribution License, which permits unrestricted use, distribution, and reproduction in any medium, provided the original author and source are credited.
Correspondence to: Antonio $\mathrm{Di}$ Ieva, MD, $\mathrm{PhD}$, Macquarie University, Sydney, NSW, Australia, E-mail: diieva@hotmail.com

Received: October 18, 2016; Accepted: December 16, 2016; Published: December 19, 2016 